Betrag der jährlichen Refraktion zuvor vergrößert worden sind (die Bemerkung des Herrn Pannekoek betreffend die angenommene Parallaxe von $0^{\prime \prime}$ Io für die Vergleichsterne beweist mir auch hier, daß er wichtige l'unkte in meiner Arbeit ubersehen hat), folgt nur in einem der vier Fälle ein negativer Wert für die jährliche Refraktion und die Beträge derselben dürften namentlich für die zwci letzten Sterne ( $\alpha$ Lyrae und $\alpha$ Cygni) durchaus reelle sein. Ein derartiges Ergebnis steht aber mit den früheren dern Sinne nach in völligem Einklang und kann als ein weiteres Zeugnis für die Wahrscheinlichkeit des Vorhandenseins einer jährlichen Refraktion aufgefaßt werden; ob dabei o"! 5 oder o"35 herauskommt, ist ziemiich gleichgiltig.

Herr Pannekoek hat schließlich die von mir noch angeführten Resultate aus den drei unvollständig beobachteten Pulkowaer Sternen, welche sich den vorigen ganz gut anschließen, überhaupt nicht mehr beachtet, obgleich die Beobachtungsgenauigkeit des Vertikalkreises auch hier von vorn. herein für die Realität der gefundenen Differenzen sprechen kann und gerade $\alpha$ Tauri als einziger Sterr: südlich von der Ekliptik, auf den somit in der Sonnenuähe die jährliche Refraktion entgegen der positiven Deklinationsrichtung wirkt, von besonderem Interesse ist.

\section{Nordhalbkugel}

Südhalbkugel

Das heißt aber soviel, daß die Gesamtamplitude (zwischen Sommer und Winter) sowohl eines aus der Parallaxe gefolgerten $z$-Gliedes, wie eines solchen, daß sich auf eine Temperaturperiode in den Neigungen der atmosphärischen Schichten gründet, für gleiche nördliche und südliche Breite gleiche Größe, aber ungleiches Vorzeichen haben wird, nur mit dem einen Unterschied, daß das letztere bei der Parallaxe von vornherein gegeben und auci mit den Beob-
Mit den vorstehenden Erörterungen glaube ich nun genügend bewiesen zu haben, daß es vorläufig um die Sache der 》jährlichen Refraktion " noch lange nicht so schlecht steht, wie Herr Pannekoek dies darstellen möchte. Im übrigen aber wiederhole ich meine frühere Bemerkung, daß hier nur die Zukunft in Gestalt einer systematisch angelegten praktischen Untersuchung Gewißh heit bringen kann (siehe meine diesbezüglichen Vorschläge) und ich gebe die Erwartung nicht auf, daß sich Sternwarten finden werden, welche mit den erforderlichen instrumentellen Mitteln auch das nötige Interesse verbinden, um zur baldigen Lösung einer für die mo. derne Stellarastronomie so wichtigen Frage beizutragen.

Es darf dabei nicht außer acht gelassen werden, daß die bereits geplante Einrichtung von Polhöhenstationen auf der Südhalbkugel keine besondere Entscheidung darüber bringen kann, ob das Kirnurasche Glied von systematischen Neigungen der Luftschichten herriuhrt - vorausgesetzt, daß diese symmetrisch zum Äquator vor sich gehen und ihre Periode ein Jahr beträgt, mit den Maxima und Minima im Sommer und Winter der betreffenden Halbkugel - oder ob es die Wirkung starker Parallaxen der Sterne (somit indirekt der jäbrlichen Refraktion) darstellt. $E s$ ist nämlich wegen der Parallaxe:

$$
\text { Basel, r } 905 \text { April } 5 .
$$

\title{
L. Courvoisier.
}

\section{Uber eine im Sonnenspektrum beobachtete Umkehr der $\mathrm{D}_{3}$-Linie. $\left.{ }^{1}\right)$}

Von Dr. H. Kreusler.

Da das Auftreten der dunklen $D_{3}$-l,inie im Sonnenspektrum augenscheinlich nicht häufig wah genommen wird, dürte vielleicht eine im vergangenen Jahre in Berlin gemachte Beobachtung dieser Art von Interesse sein.

1870 Sept. 22 hat bereits Young in der Penumbra eines Flecks $D_{3}$ als grauen Schatten gesehen, was unzweifelhaft auf eine Umkehr dieser Linie zurückzufuhren ist.

Die Wahrnehmungen von Ricco ${ }^{2}$ ), Belopolsky ${ }^{3}$ ) und Woods ${ }^{4}$, die gelegentlich innerhalb der hellen Heliumlinie eine sehr feine dunkle Linie fanden, berul en wohl weniger auf einer wirklichen Umkehr, als auf dem Umstande, daß $D_{3}$ in Wahrheit doppelt ist ${ }^{5}$ ).

$\mathrm{Ob}$ die von Young beschriebene und gezeichnete Erscheinung später wieder wahrgenommen wulde, habe ich aus der Literatur nicht ersehen können.
1904 Juni 12 zwischen $12^{\text {h }}$ und $2^{\text {h }}$ beobachtete ich die Sonne in Berlin an einem in meinem Besitz befindlichen 6 zöll. Reflektor mit einem Spektroskop, dessen Dispersion der von neun Schwefelkohlenstoffprismen von $60^{\circ}$ gleich kommt. Der Durchmesser des auf die Spaltebene projizierten Sonnenbildes betrug etwa $85 \mathrm{~mm}$, das Fernrohr des Spektroskops hatte achtfache Vergrößerung.

Zwischen dem Zentralmeridian und dem W-Rand der Sonne befand sich eine Gruppe von vier kleinen trapezartig angeordneten Flecken. In der nächsten Umgebung dieser Flecken waren die Fraunhoferschen Linien $C$ und $F$ unsichtbar, im Spektrum der Flecke selbst hell. Die beiden $D$. Linien zeigten außer der gewöhnlich beobachteten Verbreite. rung keine Anomalien (Young sah bei der vorher erwähnten Gelegenbeit die $D$-Linien in der Penumbra innerhalb der

1) Der Deutschen Physikalischen Gesellschaft vorgelegt in der Sitzung vom I. Juli 1904.

2) Comptes Rendus 102, 85 I, I $886 . \quad 3$ 3) Memorie d. Soc. d. Spettroscopisti 22, 1894.

4) Astronomy and Astrophysics I3, 159.

5) Astrophysical Journal 2, 236, I 895 . 
dunklen Verbreiterung hell). Die Linie $D_{3}$ war in den Flecken selbst nicht zu sehen, dagegen zeigte sie sich in der Um. gebung der Flecke, wo $C$ und $F$ unsichtbar waren, als dunkles, etwas verwaschenes, an beiden Enden spitz auslaufendes Band, und zwar nicht schwarz, sondern matt grau.

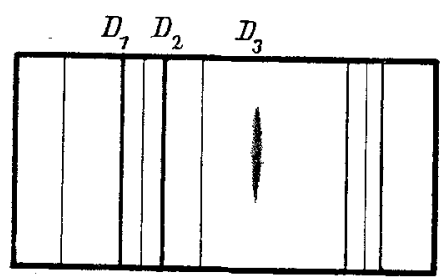

Die Erscheinung war sehr augenfällig; Herr Dr. Starke, Privatdozent am physikalischen Institut zu Berlin, der zufällig hinzukam, sah die dunkle Linie auf den ersten Blick. Leider wurde die Beobachtung durch Bewölkung vielfach unterbrochen, sodaß eine genauere Okularbeobachtung der Flecken und ihrer Umgebung, besonders mit Rucksicht auf die Anwesenheit von Fackeln nicht angestellt werden konnte.

Juni 13 war $D_{3}$ noch dunkel zu sehen, allerdings nur schwierig, weil sehr matt. Das Aussehen der Linien $C$ und $F$ war von ihrem gewöhnlichen Anblick nicht mehr verschieden. Fackeln von besonderer Helligkeit waren an diesem Tage in der Umgebung der Flecken nicht zu bemerken.

Zu erwähnen wäre noch der Umstand, daß sowohl Youngs Beobachtung 1870 in die Zeit der maximalen Fleckentätigkeit fiel, als auch, daß meine Wahrnehmung nicht lange vor dem augenblicklichen Maximum stattfand. Indessen müssen wohl erst weitere Beobachtungen lehren, ob es sich hier nur um ein zufälliges Zusammentreffen handelt, oder ob die Umkehr der $D_{3}$-Linie in engerem Zusammenhang mit den Maxima der Sonnenflecke steht.

Berlin, I 905 März 6.

H. Kreusler.

\title{
Uber die Temperatur der Sonne.
}

\author{
Von Dr. Richard Lucas, Assistent der technischen Hochschule in Braunschweig.
}

Die großen Errungenschaften, welche die Physik der hohen Temperaturen in den letzten Jahren davontrug, hat uns in den Stand gesetzt, auf verschiedenen unabhängigen Wegen das schwierige Problem der Ermittlung der Sonnentemperatur erfolgreich anzugreifen und diese Temperatur in ein relativ kleines Intervall einzuschließen. In neuester Zeit ist nun zur Bestimmung hoher Temperaturen ein Verfahren verschiedentlich ${ }^{1}$ ) angewendet worden, weiches sich durch große Einfachheit der Handhabung auszeichnet und fundamentale Bedeutung für die Beleuchtungstechnik besitzt. Das Prinzip dieser Methode besteht darin, aus der photometrischen Gesamthelligkeit auf die Temperatur des glühenden Körpers zu schließen. Während man sich zuerst damit behalf, das rapide Ansteigen der Helligkeit mit der Temperatur durch eine interpolatorische Potenzregel auszudrücken (bei Weißglut sei die Helligkeit etwa der $15^{\text {ten }}$ resp. $14^{\text {ten }}$ Potenz der absoluten Temperatur proportional) ist neuerdings durch E. Rasch das Gesetz der photometrischen Strahlung gefunden und an den experimentellen Daten geprüft worden ${ }^{2}$ ). Das Gesetz lautet:

$$
\log \text { nat } \boldsymbol{\Phi}=C-\frac{K}{T}
$$

wobei $C$ und $K$ Konstante, $\Phi, T$ Helligkeit und Temperatur in absoluter Zählung bedeuten.

Für die Gesamtlichtstärke des schwarzen Körpers bei verschiedenen Temperaturen, ausgedrückt in Hefner-Kerzen (HK) liegen folgende Daten vor:

I Quadratmillimeter schwarzer Strablung sendet aus:

\begin{tabular}{|c|c|c|}
\hline & Beobachter \\
\hline 0.0024 & $\mathrm{HK}$ & Lummer \\
\hline 0.0220 & » & und \\
\hline 0.0635 & $»$ & Pringsheim \\
\hline 0.91 & $»$ & Nernst \\
\hline
\end{tabular}

Die Helligkeit $\boldsymbol{\Phi}$ des schwarzen Körpers in HefnerKerzen pro Quadratmillimeter beträgt bei der absoluten Temperatur $T$ danach:

$$
\Phi=e^{12.943-\frac{2675^{\circ}}{T}}
$$

Berücksichtigt man nur die ersten drei Daten, da der Schmelzpunkt des Platins noch etwas unsicher ist, so ergibt sich, berechnet nach der Methode der kleinsten Quadrate:

$$
\Phi=e^{12.384-\frac{2613 \mathrm{I}}{T}}
$$

Es erscheint daher von Interesse, unter Benutzung dieses Gesetzes aus der Gesamthelligkeit der Sonne die effektive Sonnentemperatur $z u$ berechnen.

Allerdings haben nur wenige Forscher die Ermittelung der photometrischen Helligkeit der Sonne zum Gegenstand einer Untersuchung gemacht und die erhaltenen Resultate sind nicht einwandsfrei ${ }^{3}$ ). Neuerdings hat jedoch Charles Fabry ${ }^{4}$ ) in der Erkenntnis der großen Unsicherheit und Ungenauigkeit des vorhandenen Materials unter Benutzung der modernen exakten Methoden ausführliche Messungen ange-

1) Holborn und Kurlbaum, Akad. d. Wiss, Berlin 1901, 712. - Lucas, Diss. 1903, p. 8. - Nernst, Phys. Zeitschr. 4, 733 (1903).

2) E, Rasch, Annalen der Physik I4, I94 (Ig04).

${ }^{8}$ cf. G. Müller, Die Photometrie der Gestirne.

4) Comptes Rendus $87,973^{\circ}(1903)$. 